\title{
Heterogeneous, homogeneous, and enzymatic catalysis: three branches of the same scientific chapter. Introductory remarks to the "Concepts in catalysis" issue
}

\author{
Adriano Zecchina ${ }^{1}$ - Elena Groppo ${ }^{1}$
}

Published online: 14 March 2017

(C) Accademia Nazionale dei Lincei 2017

\begin{abstract}
This contribution has a fully introductive character to the issue content. It describes how the idea to connect heterogeneous, homogeneous, and enzymatic catalysis contributions took origin from a meeting of the Accademia Nazionale dei Lincei organized in Rome in 2016. In the first part of the article, the general motivations of the initiative and of the present issue entitled "Concepts in catalysis" are described. In the second part, a brief comment on the 20 contributions of this issue and on their organization is reported.
\end{abstract}

Keywords Heterogeneous - Homogeneous and enzymatic catalysis - Photocatalysis

\section{Introduction}

The Accademia Nazionale dei Lincei organized in Rome (February 25-26, 2016) an International meeting entitled "Concepts in catalysis: from Heterogeneous to Homogeneous and Enzymatic catalysis" mainly devoted to young students and researchers (mainly $\mathrm{PhD}$ and post Doc students) belonging to Italian Universities, research centers, and industrial laboratories active in heterogeneous, homogeneous, and enzymatic catalysis research. The philosophy of the meeting was based on the consideration that catalytic processes (whether they are heterogeneous, homogeneous,

Adriano Zecchina

adriano.zecchina@unito.it

Elena Groppo

elena.groppo@unito.it

1 Department of Chemistry, NIS Centre and INSTM, University of Torino, Via P. Giuria 7, 10125 Turin, Italy or enzymatic) are all based on the same principle that is the reduction of the activation barrier associated with breaking and formation of chemical bonds at the active catalytic centers. Despite this common root, heterogeneous, homogeneous, and enzymatic catalysis largely developed independently in three separate branches of science with modest reciprocal contamination. It is our opinion that there is still space for trying to overcome these barriers, at least at the level of basic knowledge and information exchange (Zecchina and Califano 2017).

Overcoming the barriers between these branches of catalysis, science was exactly the main scope of the meeting at the Accademia dei Lincei, that was designed for students belonging to the three different catalysis communities, including also the engineering and environmental and photocatalytic aspects. These ideas have guided the structure of the meeting and the character of the lectures (12), mainly based on dissemination of basic concepts in the field of catalysis, ranging from historical details to the most recent and innovative directions. The lectures content had mostly the character of small reviews with a high divulgation character. They covered different aspects of catalysis, including surface science approaches, heterogeneous catalysis, spectroscopic methods in catalysis, homogeneous catalysis, photocatalysis, quantum-mechanical methods, enzymes, and reactions engineering. The titles of the conferences are summarized below:

- Models for heterogeneous catalysts: an approach at the atomic level (H.Freund).

- Homogeneous catalysis as an important tool for organic synthesis (M. Catellani).

- Photochemistry and photocatalysis (V. Balzani).

- The discovery and evolution of metallocene-based olefin polymerization catalysts (V. Kaminsky). 
- Homogeneous and heterogeneous catalysis: bridging the gap through surface organometallic chemistry (R. Psaro).

- Heme iron centers in Cytochrome P450: structure and catalytic activity (G. Gilardi).

- Modulation of catalysis in proteolytic enzymes (M. Coletta).

- Modern electronic structure theory and catalysis: towards the simulation of complex problems in chemistry (G. Pacchioni).

- Spectroscopic methods in catalysis (S. Bordiga).

- Peculiarities and perspectives of catalytic reaction engineering ( $\mathrm{S}$. Carrà).

- Heterogeneous catalysis to realize a sustainable chemistry (F. Trifirò).

- The evolution of industrial catalysis in a complex oil refining scenario (P. Pollesel).

A not secondary scope of the meeting was to inform and convince the participants that in the last century, heterogeneous, homogeneous, and enzymatic catalysis have reached the status of true sciences, with a solid theoretical structure and a powerful legacy of experimental equipment. The profound transformations associated with this discipline evolution have led to the discovery of numerous industrial processes and the synthesis of an infinite class of new compounds, from polymers to pharmaceutical products, which have completely changed the structure of modern societies. This is even more valid today, when considering the study of supra-molecular nano-systems and biological molecules like enzymes and enzyme-inspired catalysts, a field which is gaining unprecedented importance. Furthermore, the need to observe the low concentrated and elusive intermediates typical of catalytic reactions has also fueled the development of the modern surface science methods, which are now characterized by unprecedented sensitivity.

The meeting had a high level of participation and encountered a remarkable success especially among the young researchers.

\section{A concise description and comment of the contributions of the "Concept in catalysis" issue}

On this basis, the scientific committee decided the publishing of a special issue of Rendiconti dei Lincei, which aims at reflecting the target of the meeting as indicated by the title, "Concepts in catalysis." This special issue was open not only to the plenary speakers, but also (and even more important) to the young students. This decision has been made possible by the generous help of Fondazione
Donegani and Compagnia di San Paolo. In agreement with the main purpose of the meeting of divulgating and popularizing catalysis science, most of the contributions (20) are in the form of mini-reviews devoted to beginners in the different catalysis areas. The papers can be roughly divided into six groups: surface science and heterogeneous catalysis (7), homogeneous catalysis and processes involving both heterogeneous and homogeneous catalysis (3), photocatalysis (3), enzymatic catalysis (6), and catalysis engineering (1). The partition is debatable since the various areas are strongly interwoven, as we discuss in the following.

\subsection{Surface science and heterogeneous catalysis}

Three papers of this first group, although illustrating case studies, have a very general character, in that they illustrate the problems commonly encountered in the molecular level investigation of complex heterogeneous catalysts and some of the approaches that can help in tackling the essence of these catalysts. The contribution of Freund describes a systematic approach to model heterogeneous catalysts and to characterize them at the atomic level. Two case studies are presented, $\mathrm{Au}$ islands on $\mathrm{MgO}$ for the activation of $\mathrm{CO}_{2}$ and $\mathrm{Cr} /$ silica for ethylene polymerization. These examples demonstrate that this approach may provide detailed evidence at the atomic level with an unprecedented level of accuracy, which at present cannot be attained with real catalysts. The paper of Pacchioni reports a Density Functional Theory Study of the influence of particle size on the structure, surface properties, and reactivity of catalyst nanoparticles (in this case $\mathrm{ZrO}_{2}$ ). As the illustrated methodology can be applied to other metal-oxide catalysts, this contribution has a very general character and can be of great utility for beginners in the field. Finally, the paper of Lazzarini is entirely devoted to the description of high surface area activated carbons, which is another class of materials largely employed in heterogeneous catalysis. The contribution is a good example of the successful application of a very large number of techniques, such as Solid state-nuclear magnetic resonance, X-ray powder diffraction, Raman spectroscopy, X-ray photoelectron spectroscopy, inelastic neutron scattering, and FT-IR spectroscopy to characterize the structure and the surface properties of this class of materials. The very general message coming from these three contributions is that the full characterization of a heterogeneous catalyst in all its aspects very often requires the use of a multi-technique approach, comprising both experimental and theoretical methods, and a balanced mixture of competencies and skills.

This is further demonstrated by the paper of Piovano, which faces the very old and challenging problem of 
determining the structure of single-site catalysts. The authors propose the synthesis and characterization of a titanium chloride-based, silica-supported, Ziegler-Natta catalyst for ethylene polymerization. This paper is closely connected to the Freund contribution, at least for the part concerning the structure of the silica-supported Phillips catalyst.

The contribution of Frediani deals with the activity of palladium nanoparticles supported on a polymeric material (2,2'-bipyridine end-functionalized poly(lactic acid)poly( $\varepsilon$-caprolactone) copolymer). Its relevance in this special issue is related to the fact that it elegantly demonstrates the important role of the support in determining the selectivity of the metal particles in phenyl acetylene hydrogenation. The role of surface ligands entering the coordination sphere of the active centers is highlighted.

Finally, the contributions of Trifirò and Garbarino tackle the problem of biomass exploitation by means of heterogeneous catalysis for the production of important chemical intermediates and for the production of synthesis gas and renewable hydrogen. The Trifirò paper, which has the character of a review, analyzes a series of results obtained in the production of acrolein and acrylic acid from biomass using different catalysts having mainly a mixed-oxide character. The Garbarino paper presents a technological perspective of Ni-based catalysts in the development of both gasification and gas cleanup technologies, with particular attention to the interaction of different tars molecules present in tar mixture and to catalyst deactivation by coke deposition and sulfur poisoning. The general message from both papers is that for the effective development of these technologies, several challenges have been still to be undertaken and fundamental research to optimize the processes is necessary.

\subsection{Homogeneous catalysis and processes involving both heterogeneous and homogeneous catalysis}

The Kaminsky contribution deals with a class of homogenous catalysts for olefin polymerization (metallocenes) that occupies a prominent position in the history of homogeneous catalysis. It illustrates how these catalysts allow the synthesis of isotactic, isoblock, syndiotactic, stereoblock, or atactic polymers, as well as polyolefin composite materials with superior properties. These homogeneous single-site catalysts compare very well with heterogeneous Ziegler-Natta catalysts (see the Piovano contribution), but have the advantage to provide a better understanding of the mechanism of the olefin polymerization.

The contribution of Ragaini, which summarized the results obtained by the authors in the last 30 years, is focused on the reduction of suitably substituted organic nitro compounds by $\mathrm{CO}$, catalyzed by different metal complexes. A special attention is given to the more recently developed inter-molecular reactions.

Finally, the Di Serio contribution discusses the most important results and the still open challenges in the production of biodiesel from waste oils (characterized by high concentrations of Free Fatty Acids) using basic catalysts. This paper is strongly related to the problem of biomass utilization treated by Trifirò and Garbarino contributions. It is considered here because homogeneous and heterogeneous processes are compared.

\subsection{Photochemistry and photocatalysis}

The Ceroni and Balzani paper is a mini-review on the concepts of photochemistry and photocatalysis. The contribution is designed on the idea that in order to understand the potential applications and the working mechanisms of photocatalysis, it is necessary to know some important concepts of photochemistry, the branch of science that deals with the interaction of light and matter. The concept of photosensitization that may take place either in homogeneous or heterogeneous phase is then illustrated. Water splitting using sunlight and suitable photosensitizers and catalysts (artificial photosynthesis) is perhaps one of the most thoroughly investigated chemical processes. Breakthrough in this area can contribute to solve the energy and climate crisis, but substantial technological development is still needed.

The contribution of Mino is focused on the use of FT-IR spectroscopy to study reactions occurring on the surface of a photocatalysts under light irradiation and to investigate the vibrational spectrum of labile intermediates. A concise overview of the most used acquisition modes (transmission, ATR and DRIFT) and the available experimental setups is provided. The selected examples highlight the potential of IR spectroscopy in monitoring the evolution of reaction intermediates and products at the photocatalyst surface. This approach, which allows to understand the reaction mechanism and to derive the kinetic laws expressed in terms of surface concentrations, might be of general interest for researchers involved in heterogeneous photocatalysis.

Finally, the contribution of Compagnoni et al. describes two different reactor configurations exploiting solar energy for two challenging processes, nitrate/ammonia abatement and $\mathrm{CO}_{2}$ photoreduction, focusing the attention on some critical operating process parameters. As for $\mathrm{CO}_{2}$ photoreduction is concerned, the study illustrates an innovative high pressure reactor that could overcome some limitation of this important process. 


\subsection{Enzymatic catalysis}

In this group of contributions, the four papers coming from the Gilardi group are dominating and have all a review character. Two of them can be described together, because they are both related to the cytochromes P450 superfamily. This family of enzyme comprises members distributed in all domains of life, where they participate to the metabolism of endogenous and exogenous compounds. The first paper of this series deals with the structure and catalytic activity of the heme iron centers. These enzymes can perform a series of oxidative reactions on a broad range of chemically different substrates and, for this reason, they are optimal candidates for biocatalytic purposes and, in general, technological applications. These enzymes have inspired the synthesis of iron-based homogeneous catalyst mimicking the natural catalysts. The so called catalytically self-sufficient cytochromes P450 belong to this superfamily and are discussed in the second paper of the series. These enzymes present all the advantages of the regio- and stereoselectivity of typical P450s combined with the fact that, due to the natural fusion with their reductases, their application in biocatalysis does not need the addition of other proteins: only $\mathrm{NAD}(\mathrm{P}) \mathrm{H}$ and substrate are needed to trigger catalysis.

In a subsequent contribution, $[\mathrm{FeFe}]$-hydrogenases are illustrated. These enzymes have been studied extensively because of their potential exploitation in $\mathrm{H}_{2}$ production for clean energy storage. [ $\mathrm{FeFe}]$-hydrogenases have been used in several fermentative approaches where microorganisms are engineered to convert low cost materials (e.g., wastes) into $\mathrm{H}_{2}$. $[\mathrm{FeFe}]$-hydrogenases have also been proven to be excellent catalysts in different in vitro devices that can produce hydrogen directly from water, either via water electrolysis or via light-driven mechanisms, thus allowing the direct storage of solar energy into $\mathrm{H}_{2}$.

In the last contribution, the Class $\mathrm{B}$ flavoprotein monooxygenases, especially Baeyer-Villiger enzymes, are discussed. These enzymes are highly selective in chemo-, regio-, and enantio-selective oxygenation reactions and are useful in the synthesis of high-value chemicals. This minireview provides an overview of some stereo-selective reactions carried out by Class B flavoprotein monooxygenases and the efforts made in order to make these biocatalysts suitable for industrial applications.

The contribution of Ascenzi summarizes the properties of human serum albumin (HSA), the most abundant protein in plasma that displays several functions including heme transfer. In particular, ferric human serum heme-albumin (HSA heme) and ferrous HSA heme catalyzes the conversion of nitrite to nitrogen monoxide. The drug-dependent five-to-six coordination transition of the heme-Fe atom modulates the catalytic activity.
The structure and activity of an enzyme belonging to the protease family, the prostate-specific antigen, is reviewed in the Coletta contribution. Proteases are a class of enzymes that lower the activation energy for the cleavage of the peptide bonds by polarizing the carbonyl group. The active center of the enzymes is not containing transition metal ions and hence they represent an example of so called organic catalysis. The $\mathrm{pH}-$ dependence of the catalytic parameters clearly indicates the existence of protonation/deprotonation processes involving (at least) two ionizing groups in the proximity of the active site. The importance of this catalyst for the treatment of prostatic diseases is self evident.

\subsection{Catalysis engineering}

The industrial aspects of catalysis engineering are illustrated in the Carrà contribution. The importance of a contribution in this area derives from the fact that catalytic reaction engineering offers a rational approach to quantify the simulation, design and performance of the industrial catalytic processes. The involved methods follow a multiscale approach that spans from molecular size to the reactor scale. The biocatalysis that deals with microorganisms and enzymes, can be considered a special case of heterogeneous catalysis and therefore the simulation and control of the involved reactors is faced starting from the basic knowledge on the enzymatic kinetics and cell growth dynamics. This approach confers to the Carrà contribution a very general and tutorial character.

\section{Conclusions}

From the concise illustration concerning the content of the 20 contributions of this issue, it can be concluded that the initial idea to cover the most important areas of catalysis science has been fulfilled. Of course we are fully aware of the limitations of the issue content particularly when the ambitious and visionary character of the initiative is taken into account. We only hope that the general message concerning the necessity to widen the approach to catalysis science can be appreciated.

\section{Reference}

Zecchina A, Califano S (2017) The development of catalysis. A history of key processes and personas in catalytic science and technology. Wiley, Hoboken (in press) 